\title{
AN EASILY EXTENDABLE INTERPRETER COMPRISING MCA AND CAMAC COMMANDS
}

\author{
E.L. BAKKUM and R.J. ELSENAAR \\ Fystsch Laboratorium, Rijksumversitett, Princetonplein 5, P.O. Box 80.000, 3508 TA Utrecht, The Netherlands
}

Recelved 2 Aprıl 1984

\begin{abstract}
A BASIC interpreter is a useful tool for writıng small programs in a quick and easy way. For the control of experiments, however, It lacks a number of essential features. A BASIC-like command interpreter BACO has therefore been developed. It runs on PDP-11 computers with the RSX-11M operating system. Its major advantages over BASIC are: (1) new FORTRAN routınes can be implemented simply, and (2) interrupts can be processed at interpreter level.

As an application the implementation is described of routınes to control a CAMAC system and of a multichannel analyzer simulation. The CAMAC commands follow the line proposed by the ESONE committee. Since an interpreter is inherently rather slow, the commands are intended for moderately fast data transfer and interrupt handling, which suffices for the control of many experıments
\end{abstract}

\section{Introduction}

It is impossible to write one single program satisfying the specific needs of the numerous types of nuclear physics experiments. On the other hand the different experiments have so many software requirements in common, that writing a separate program for every application easily leads to duplication of features in many dedicated programs. Therefore it is advantageous to have a program that carries out a number of the common tasks and that can be extended with all the dedicated routines required for a specific kind of experiment.

One of the common tasks is the interpretation of commands, which suggests the use of a BASIC interpreter. Since BASIC, however, is more directed towards off-line programs, the experimental applications gave rise to the development of the new command interpreter BACO [1,2]. The interpreter is used as a basis for a number of programs, thereby assuring that the general commands are equally well recognized by all programs, and that differing commands anyhow have the same syntax. This is especially convenient for inexperienced computer users.

BACO performs the following tasks:

- it accepts and decodes the commands, which are of a simple BASIC-like format; several standard commands facilitate the control of the experiment,

- it intercepts interrupts issued by the experiment and coordinates interrupt processing according to commands defined beforehand,

it enables easy implementation of FORTRAN routines for special applications.
The BACO interpreter is used on the three PDP-11 computers of our department. In nuclear physics large amounts of data are produced. For storage and preliminary analysis of these data, a minicomputer is very well suited, whereas further analysis is performed on a larger system. The off-line data analysis and program development is carried out on a PDP-11/70 computer, while two PDP-11/34A minicomputers are used for data acquisition and measurement control. The PDP$11 / 70$ requires a multi-user operating system to deal with its more than 30 terminals. In order to provide maximum compatibility between the three computers, they are all run with the multi-user operating system RSX-11M V4.0. This has the important advantage that tasks developed and tested on the PDP-11/70 can be transported as taskfile to a PDP-11/34 and run immediately without any adaptation. In addition, RSX$11 \mathrm{M}$ has many features not available in smaller operating systems. The main disadvantage is the space requirement of the operating system (in our case about $32 \mathrm{k}$ words). The minicomputers thus need more memory than an operating system like RT11. To meet the requirements of all our present applications, the PDP$11 / 34$ 's are supplied with $124 \mathrm{k}$ words of memory (the maximum for these computers). For many applications, however, about half of this would be sufficient.

At present BACO is extensively used in our department as the basis for three programs. The Spectrum Analysis Program SAP [3] is usually running at the PDP-11/70. The second, called SPECTR [4], runs at the PDP-11/34A computer used for experiment control at the $6 \mathrm{MV}$ tandem Van de Graaff accelerator. SPECTR controls the event-mode data collection via a home-built 


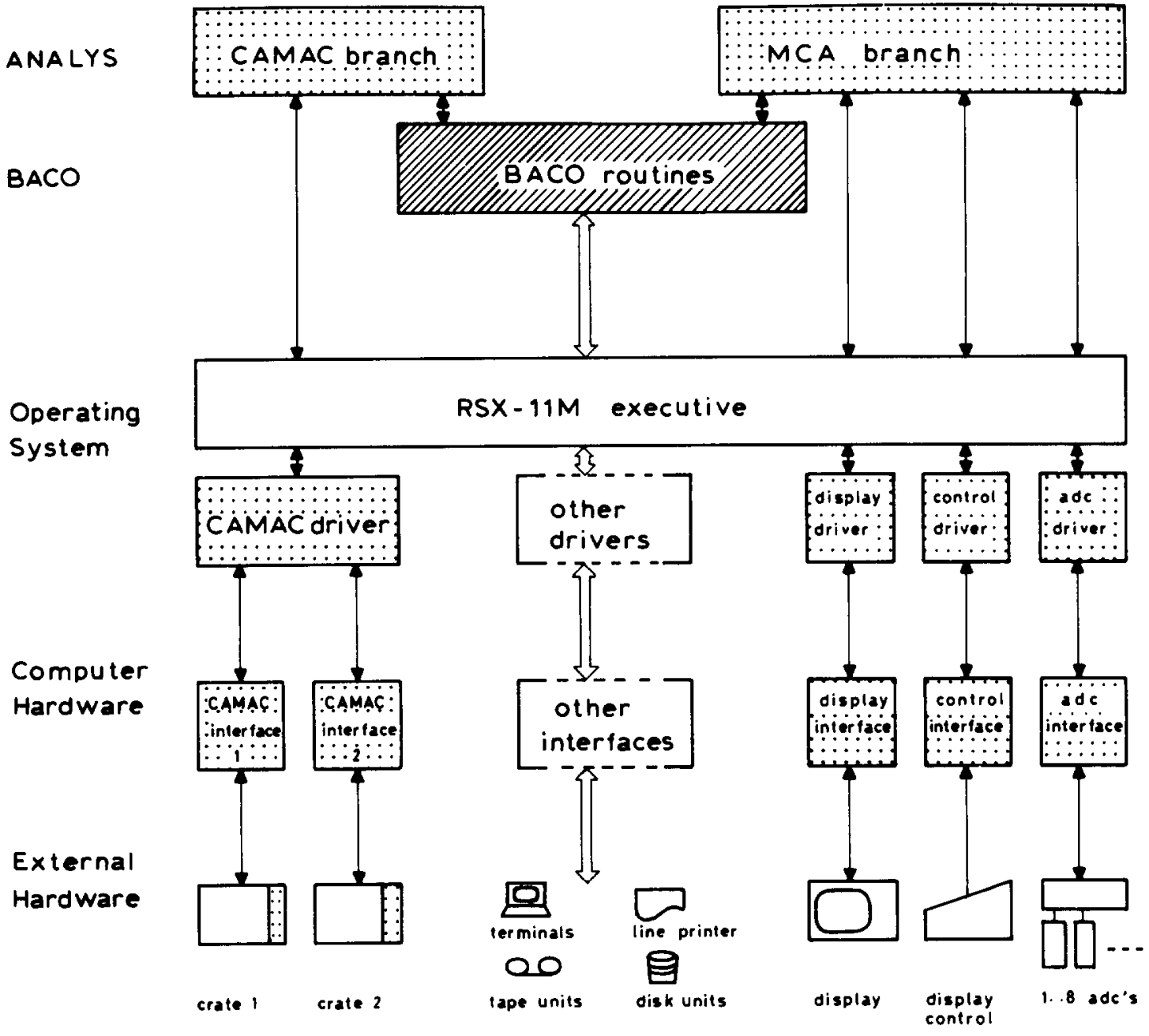

soft/hardware dedicated to the application

W the BACO system which the programmer can conceive as an extension of the Operating System

\section{standard soft/hardware}

Fig. 1. The structure of the measuring program ANALYS.

interface or via a CAMAC system. The same program is also used for the off-line sorting of event-mode data on the PDP-11/70 computer. The third program, ANALYS, is described in this paper as an example of BACO and the CAMAC interfacing. This on-line measuring program, in use at the $3 \mathrm{MV}$ Van de Graaff accelerator, serves two purposes:

- it provides a programmable multichannel analyzer (hence its name),
- it supplies an advanced set of CAMAC commands for control of the measurement by setting and readout of parameters, via two CAMAC crates.

These two tasks are performed via a set of subroutines. They constitute two branches connected to the BACO basis and via the Operating System to the corresponding hardware. The interaction is schematically indicated in fig. 1 . 


\section{Properties of the BACO command interpreter}

An experiment is controlled by commands typed in on the terminal, like "CLEAR 1" to clear spectrum number 1 . BACO interprets these commands, evaluates the arguments if present (in the above example the argument is the spectrum number) and transfers them to the appropriate routine that performs the task: in this case the array containing the specified spectrum is set to zero's.

The commands can be executed immediately after being typed in, but they can also be loaded to constitute a "control program" for later execution. The syntax of such a program very much resembles that of the language BASIC, from which the name BACO is deduced: BAsic COmmand interpreter.

A control program may contain commands to evaluate arguments for a routine or also to determine whether a call to the routine is in fact required. The following example gives an impression of such a program:

$$
\begin{aligned}
& \text { \#10 LET SPEC }=0 \\
& \text { \#20 IF }(\text { CONTENT }(\text { SPEC } 1)>10000) \text { THEN LET } \\
& \text { SPEC }=1 \\
& \# 30 \text { IF }(\text { CONTENT }(\text { SPEC } 2)>10000) \text { THEN LET } \\
& \text { SPEC }=2 \\
& \text { \#40 IF }(\text { SPEC }>0) \text { THEN CLEAR SPEC }
\end{aligned}
$$

The example shows four command lines preceded by line numbers, which serve to determine the order in which the commands will be executed. With the aid of BASIC-like commands ("LET", "IF") and of dedicated routines (the function "CONTENT"), a parameter is evaluated ("SPEC") which serves as argument for another dedicated routine ("CLEAR").

During the measurement the computer will collect data for a preset time, or until the experiment itself generates a so-called interrupt, e.g. triggered by the collection of a preset amount of beam charge on the target. As a consequence of the interrupt a previously declared part of the control program (a subroutine) is executed in order to read out the data, to store the results on disk or magnetic tape, and to reset and restart the measurement.

The properties of BACO relevant to its application in on-line control programs are summarized as follows:

1. BACO contains most BASIC features.

2. Commands and syntax are easily learned and handled, as in BASIC.

3. Contrary to BASIC, implementation of new dedicated FORTRAN routines and variables is easy.

4. A running control program can be interrupted by the experimentalist, e.g. for checks or for corrections. The program then continues where it was interrupted; it is thus not required to start from the very beginning.

5. The program can delay itself for a specified time, or until the occurrence of an interrupt.

6. A set of commands (a subroutine) can be specified that will be executed after an interrupt.

These properties are elaborated in the following sections.

\subsection{A versatule interpreter}

Most BASIC commands have a BACO equivalent, albeit that the syntax is slightly different. Examples are the statements LET, IF..., FOR... NEXT, GOTO, CALL, RUN, etc. Much attention is paid to the input from and output to terminal and file. The PRINT statement e.g. will of ten be used to show results or parameter settings. These can easily be listed in table form with the aid of FORMAT specifiers. Texts, numbers, or even expressions can be read in with the INPUT statement.

Commands and control programs can also be fetched from file at the moment they are needed. This gives a limited overlay capability and thus enables large control programs.

\subsection{An easy syntax}

Experimentalists usually prefer short and easy commands over syntactical perfection. Although an interpreter can check a BASIC statement, like LET L1\% = L\% + LEN(T\$), faster for type conflicts etc., the BACO equivalent $\mathrm{LET}$ LTOT $=\mathrm{L}+\mathrm{LENGTH}($ TEXT) is simpler to read, and thus reduces the risk of typing errors. $\mathrm{BACO}$ variables are identified by names consisting of up to six alphanumerical characters, and the type of a variable is determined by the type of the value assigned to it. Thus the type of the variable $R$, resulting from the expression

\# LET $\mathrm{R}=\mathrm{X}+\mathrm{Y}$

is "number" if $X$ and $Y$ are numbers, and is "string" if $X$ and $Y$ are strings (the addition operation between strings is defined as concatenation).

\subsection{Easy implementation of new FORTRAN routınes}

When a new routine is implemented, the interpreter has to recognize the corresponding command. After the addition of a new FORTRAN routine named CLEAR (which clears a spectrum) with the integer argument I (denoting the number of the spectrum to be cleared), BACO should translate the command

\section{\#CLEAR I}

to the FORTRAN subroutine call

\section{CALL CLEAR(I)}

The link between the command and the FORTRAN routine is made via a file that is accessed when the 
BACO interpreter task is built. The name of the command and the type of its arguments are defined by the addition of a single line:

\section{FORTRAN CLEAR INTEGER}

The line states that BACO should recognize the command "CLEAR" by which the FORTRAN subroutine with the same name is called and that it has an argument of the type "INTEGER".

With respect to the implementation of FORTRAN functions BACO has the following characteristics differing from those of many BASIC interpreters:

- A command referring to a user implemented subroutine has the same syntax as a standard BACO command. Whereas the BASIC call reads:

500 CALL "CLEAR" (I)

the BACO equivalent is:

\section{\# 500 CLEAR I}

without the additional word "CALL", and without quotes or parentheses. It has the same syntactical structure as a reference to a standard BACO routine like PRINT in e.g.

\section{\#510 PRINT I}

- The arguments can be "called-by-value" as well as "called-by-name". The first method is used when the subroutine needs the value of an argument, while the latter case enables the subroutine to return a value, calculated within the subroutine.

- A function can be implemented in the same way as a subroutine. The result of a function can only be a number or a logical, as FORTRAN does not allow other types.

- It can be useful to make some variables accessible by both the FORTRAN routine and by the BACO user. Therefore variables in a FORTRAN common partition can be defined to be recognized also as BACO variables.

\subsection{Interruption of a running program via the terminal}

A program in BACO consists of a set of statements each preceded by a line number. Normally the program lines are executed sequentially, and the BACO interpreter will only accept a new command after completion of the program. A faulting program could thus be stopped only by the complete abortion of the interpreter task via an MCR command. This could result in loss of data and improperly closed files. To avoid this, a running BACO program can be interrupted with the MCR command

\section{$>$ INT}

which activates the small task "INT" that directs BACO to suspend the execution of the control program and to accept commands from the experimentalist.

After the intervention BACO will continue with the statement where the interruption occurred.

\subsection{The delay option}

After the command

\# WAIT 〈sec $>$

the BACO task will delay the execution for $\langle\mathrm{sec}\rangle$ seconds. After that it resumes with the next statement. This command is useful in a control program to enable a measuring device to collect data. In the case of the multichannel analyzer simulation described below, the command is used to stop BACO durung the (DMI) accumulation of a spectrum, as this does not require any software intervention.

It should be mentioned that the execution can also be restarted by an interrupt, so that the statement can be used to wait for such an interrupt.

\subsection{Interrupt handling}

An interrupt can occur at an arbitrary moment, and it requires the immediate execution of a set of commands. The user can specify a routine which contains these commands. When an interrupt occurs, first the current line of the running control program is finished. Then the program is temporarily stopped and the specified routine is executed. After that, execution of the main program resumes at the point where it was interrupted (see also section 4.2.3).

\section{The analyzer simulation}

The BACO interpreter described above constitutes the framework for the implementation of application oriented routines. The ANALYS version thus simulates a programmable multichannel analyzer with all the usual analyzer features. For the interfacing of the ADCs to the computer, several concepts are generally followed, often involving the use of more or less intelligent CAMAC modules $[5,6]$. We have chosen for a direct coupling to the computer via two kınds of home built interfaces. One is developed for event mode data collection and is used by the program SPECTR [4]. The other interface is applied with the accumulation of singles spectra and is controlled by the program ANALYS. The last interface consists of the following components:

- A fast Direct-Memory-Increment interface to link up to eight 8192-channel ADCs to the computer, which can handle a total count rate of up to 120000 per second.

- A video screen, which displays a (part of the) spectrum selected by the experimentalist.

- A number of additional keys to change the displayed part of the spectrum and the ROI (Region-Of-Interest).

All three hardware parts have their own software driver, 
so that programs can communicate with them in a standard RSX-11M manner (see fig. 1).

The analyzer simulation contains the following features:

- As many spectra can be defined as will fit in the available memory of the computer. Each channel has a maximum content of $2^{24}-1$ counts.

- Commands are available to display (part of) a spectrum, to clear a spectrum, and to change or read the content of a channel.

- The lower and the upper limits of the displayed part can be read out by the interpreter, which enables the user to program any number of ROIs.

- Spectra can be dumped on and restored from tape.

\section{Implementation of CAMAC features}

As $\mathrm{BACO}$ is developed as an interpreter for measurement control, it forms a excellent framework for extension with CAMAC routines. The obvious problem inherent to CAMAC control via an interpreter is its slowness. To circumvent this problem one can decide to control CAMAC via a high level language like FORTRAN and develop a set of routines to facilitate this $[7,8]$. This, however, forces the user to write control programs in FORTRAN, while it is much easier and faster to program a BASIC-like interpreter, especially for people unexperienced with computers. Our aim therefore was minimalization of the slowness by an intelligent home-written CAMAC driver [9] which can act fast on the basis of presetted commands. For ANALYS however, this is of little importance because only slow processes like presetting and reading out of counters and the positioning of the detector, are allotted to CAMAC.

\subsection{Hardware and system-end of the software}

Our CAMAC systems contain the Nuclear Enterprise 9030 controller, which is coupled to the PDP 11 Unibus in one computer via the NE interface, and in the two others via a home-built serial interface based on a BB11H with opto-couplers. As programs communicate with CAMAC via the driver, the use of other controllers requires only adaption of this driver and no changes in the BACO task itself (see fig. 1). The driver concept has the following additional advantages:

- The CAMAC driver follows the line of other RSX$11 \mathrm{M}$ devices. Communication with it thus proceeds via the standard QIO calls and other EXECUTIVE features, either as assembler macro's or as FORTRAN subroutine calls. Consequently the communication with CAMAC is much more transparent for the FORTRAN programmer.

- A CAMAC crate can be accessed simultaneously by more than one task, and even by more than one user, with the possibility of linking specific LAMs (LookAt-Me interrupts) to specific tasks.

\subsection{Syntax of the CAMAC commands}

For the format of the CAMAC commands the recommendation for a CAMAC extension to a BASIC interpreter [10] is taken as a guideline, although some differences are induced by the existing BACO syntax.

The most important CAMAC commands are demonstrated in the following example (a presettable counter).

\subsubsection{Declaring a module}

Before a CAMAC module can be accessed, it should be declared with the command:

$$
\begin{aligned}
& \text { \#DECLARE }\langle\text { name }\rangle \text { CAMAC }(\langle\mathrm{A}\rangle\langle\mathrm{N}\rangle\langle\mathrm{C}\rangle) \\
& (\langle\mathrm{Fr}\rangle\langle\mathrm{Fw}\rangle)(\langle\mathrm{Cr}\rangle)(\langle\mathrm{Cw}\rangle)(\langle\mathrm{GL}\rangle)
\end{aligned}
$$

Here 〈name〉 is the formal name to be used from now on in commands referring to the module. The symbols $\langle A\rangle,\langle N\rangle$ and $\langle C\rangle$ stand for the subaddress, station number and crate number, respectively, where the module can be found, while $\langle F r\rangle$ and $\langle F w\rangle$ indicate the read- and write-access functions. The parameters $\langle\mathrm{Cr}\rangle$ and $\langle\mathrm{Cw}\rangle$ are one or more data conversion specifiers for read and write, respectively. $\langle\mathrm{Cr}\rangle$ is specified if the data of the CAMAC modules are coded in, e.g., one's complemented or binary-coded-decimal format. In these cases $\langle\mathrm{Cr}\rangle$ should be "COM" or "BCD", respectively, which results in automatic decoding upon a read reference to the module. $\langle\mathrm{CW}\rangle$ is used correspondingly for a module expecting input in coded format. The parameter $\langle G L\rangle$ is the (graded) LAM number associated with an interrupt from the specified module (normally its station number). If a default value suffices, the corresponding parameter can be omitted.

If one wishes to use the formal name SCALER to refer to a counter located at station 4 and subaddress 1 . which produces data in binary-coded-decimal format, and uses default settings for the remaining parameters, the declaration would be:

\# 100 DECLARE SCALER CAMAC $\left(\begin{array}{ll}1 & 4\end{array}\right)\left(\begin{array}{l}\text { )(BCD) } \\ \text { (BC }\end{array}\right.$

\subsubsection{Referring to a module}

A module can be accessed by simply using its formal name in an expression, just like the name of any other variable. The content of the counter can thus be read by:

\#150 PRINT "counter content is:",

SCALER/1000, "kCounts"

and preset by the normal BACO assign statement:

\#200 LET SCALER $=1000$

Some operations require a reference to a module 
already declared with a different subaddress or a different function. This is possible, but it will not be discussed here for the sake of brevity.

Actions without data transfer are carried out with the CONTROL command:

\# CONTROL<name $>$ control-function $\rangle$

Here <control-function $\rangle$ is the standard CAMAC function code $F\langle n\rangle$ for the control action. For most CAMAC functions BACO has already a symbolic name, For example, clearing the counter can be done by:

\section{\#250 CONTROL SCALER CLEAR1}

\subsubsection{Interrupts}

The counter in the present example is assumed to send a LAM signal to the computer after receiving a preset number of pulses. This interrupt should cause the execution of a certain subroutine. The LAM and the subroutine are connected by the statement



The number <line-number > points to the first line of the subroutine. In the current example this could be:

\section{\# 300 ON SCALER \#350}

The subroutine beginning with statement $\# 350$ could be used to clear the LAM:

\section{\# 350 CONTROL SCALER CLRLAM \#360 RETURN}

To summarize the example, the consequences of an interrupt issued by the counter are:

- the current commandline is finished,

- the commands beginning at line \#350 are processed,

- after the RETURN statement (at line \#360), execution of the main program is resumed with the command line following the one that was interrupted.

\section{Conclusion}

The command interpreter BACO runs with the RSX$11 \mathrm{M}$ multi-user system and is especially designed for use in physics experiments. The interpreter resembles BASIC with respect to the syntax and the available commands. The commands thus are simple, and further simplification results from an adjustment of the BASIC syntax which improves readability.
BACO supplies a framework that has to be completed with dedicated FORTRAN routines to match the applications. The available facilities make it very easy to implement those routines. The combination of a set of routınes simulating a multichannel analyzer is described as an example.

An experiment frequently demands program action via an interrupt. The user can define a routine that starts up when the interrupt occurs. This feature and the structure of BACO itself make it very well suited for the implementation of a set of CAMAC routines applicable for moderately fast experiment control functions.

More details of BACO are described in the extensive manual [11], which is available on request.

This work was performed as part of the research program of the "Stichting voor Fundamenteel Onderzoek der Materie" (FOM) with financial support from the "Nederlandse Organisatie voor Zuiver-Wetenschappelijk Onderzoek" (ZWO).

\section{References}

[1] J.B. van Meurs and E. Lopez Cardozo, Software Pract. Exp. 7 (1977) 85.

[2] J.B. van Meurs, F.P. Boomstra, E. Lopes Cardozo, C.M. Prins and K. Straatman, BACO manual, internal report (1977).

[3] F.P. Boomstra, C.M. Prins and B. Rector, Annual Report of the Robert J. Van de Graaff Laboratory, Utrecht (1977) p. 28, and SAP manual, internal report.

[4] R.J. Elsenaar, Annual Report of the Robert J. Van de Graaff Laboratory, Utrecht (1982) p. 64, and SPECTR manual, internal report (1982).

[5] Y. Nagashima, H. Kimura and K. Kuriyama, Nucl. Instr. and Meth. 206 (1983) 147.

[6] A.J. de Raaf, Nucl. Instr. and Meth. 163 (1979) 313.

[7] Ö. Ciftcioglu, Nucl. Instr. and Meth. 174 (1980) 21.

[8] R. Feenstra, R.R. Johnson and C. Winter, Nucl. Instr. and Meth. 160 (1979) 511.

[9] R.J. Elsenaar, Annual Report of the Robert J. Van de Graaff Laboratory, Utrecht (1982) p. 64.

[10] ESONE (European Standard Of Nuclear Electronics) Committee, REAL-TIME BASIC for CAMAC, ESONE/RTB/01 (1976).

[11] E.L. Bakkum, BACO manual, internal report (1983). 